\title{
Seed Treatments and Storage Containers on Storability of Petunia Seeds
}

\author{
K. Natarajan* \\ Seed Science \& Technology, KVK, Virudhachalam, Tamil Nadu, India \\ *Corresponding author
}

\section{A B S T R A C T}

\section{Keywords}

Petunia, Seed, Seed treatments, Containers, Storability

Article Info

Accepted:

10 May 2019

Available Online:

10 June 2019
Studies were made to evaluate the seed treatments (halogen mixture, bavistin and diflubenzuron) and storage containers (paper bag, 700 gauge polyethylene bag and aluminium foil polylaminated pouch) on storability of petunia seeds with cv.Mix. The results revealed that the germination reduces from 96 to 75 per cent with 12 months of storage period under ambient condition irrespective of treatments. Among the seed treatments, halogen mixture followed by diflubenzuron found to be better as seed treating chemicals. Storability of seed is better in moisture vapour proof container (aluminium foiled pouch) than moisture pervious container (paper bags).

\section{Introduction}

On account of the diverse biological activities taking place in seed during storage, the seed deteriorates in quality, resulting in impairment of germination and vigour (Copeland, 1988). Storability of seeds is determined by vide number of factors viz., period of storage (Delouche and Baskin, 1973) type of containers (Bhattacharya et al., 1982), initial seed quality (Maranville and Clegg, 1977), storage environment (Justice and Bass, 1978), seed moisture content (Roberts, 1986) and seed treatment (Basu and Rudrapal, 1980). Among these, prestorage seed treatments and packaging materials had their pronounced impact on the storability in view of their protection rendered against the biotic factors and irreversible deterioration (Barros et al., 1983; Burris, 2002). In prestorage seed treatment, fungicides and insecticides play wider role. During the past two decades the development of third generation pesticides, the insect growth regulators (IGRs), are being introduced as insect control agents (Elek and Longstaff, 1994). The term IGR is coined to include all those compounds that disrupt growth and development of insects. Unlike organophosphorus, carbonate and other chemical insecticides carrying actual and/or potential risks of high toxicity to mammals 
and environmental pollution hazards, IGRs appear to be effective against target insect with relative safety to mammals and the environment (Gupta, 2001). On the other side, bavistin the systemic fungicide is widely recommended for the control of seed borne pathogen and storage fungi. But Basu and Rudrapal (1980) and Dharmalingam et al., (2000) recommended halogen mixture as an economic and ecofriendly product for seed treatment. Hence to evaluate the efficacy of these chemicals on the storability of petunia seeds the present study was formulated with cv. Mix.

\section{Materials and Methods}

Freshly harvested seeds of petunia cv. Mix were dried to 8 per cent moisture content and were treated with halogen mixture @ $3 \mathrm{~g} \mathrm{~kg}^{-1}$ seed (dry dressing), bavistin @ $3 \mathrm{~g} \mathrm{~kg}^{-1}$ (slurry treatment) and diflubenzuron @ 1 ppm (slurry treatment) and were packed in paper bag, 700 gauge polyethylene bag and aluminium foil polylaminated pouch along with untreated seed and stored under ambient conditions of Coimbatore (Coimbatore (28 \pm $2^{\circ} \mathrm{C}$ : $\mathrm{RH} 70 \pm 5 \%$ ) for a period of 12 months. The experiment was formulated adopting FCRD with four replications and at trimonthly intervals the samples were collected from each treatment and replications and the seed and seedling quality parameters viz., moisture content (\%) (ISTA, 1999), germination (\%) (ISTA, 1999), root and shoot length $(\mathrm{cm})$, drymatter production $(\mathrm{mg} 20$ seedlings $^{-1}$ ) and vigour index (Abdul Baki and Anderson, 1973) were evaluated. The data gathered were scrutinized as per Gomez and Gomez (1984) for understanding the level of significance. Freshly harvested seeds of petunia cv. Mix were dried to 8 per cent moisture content and were treated with halogen mixture @ $3 \mathrm{~g} \mathrm{~kg}^{-1}$ seed (dry dressing), bavistin @ $3 \mathrm{~g} \mathrm{~kg}^{-1}$ (slurry treatment) and diflubenzuron @ 1 ppm (slurry treatment) and were packed in paper bag, 700 gauge polyethylene bag and aluminium foil polylaminated pouch along with untreated seed and stored under ambient conditions of Coimbatore (Coimbatore $\left(28 \pm 2{ }^{\circ} \mathrm{C}: \mathrm{RH}\right.$ $70 \pm 5 \%$ ) for a period of 12 months. The experiment was formulated adopting FCRD with four replications and at trimonthly intervals the samples were collected from each treatment and replications and the seed and seedling quality parameters viz., moisture content (\%) (ISTA, 1999), germination (\%) (ISTA, 1999), root and shoot length (cm), dry matter production (mg 20 seedlings $^{-1}$ ) and vigour index (Abdul Baki and Anderson, 1973) were evaluated. The data gathered were scrutinized as per Gomez and Gomez (1984) for understanding the level of significance.

\section{Results and Discussion}

Highly significant results were obtained due to seed treatments, storage period and storage containers for all the evaluated seed quality parameters except for moisture content due to treatments and root length due to containers. The moisture content of the seed which plays a prime role in seed deterioration (Copeland and Mcdonald, 1995) increased with advances in storage period and recorded the maximum of 8.3 per cent after 12 months of storage period irrespective of seed treatments and storage containers. But the increase was upto 8.6 per cent in seeds stored in paper bag due to the attainment of equilibrium moisture content with the given atmosphere (Justice and Bass, 1978). The increase of moisture content in aluminium foil polylaminated pouch and 700 gauge polyethylene bag was nil with advances in storage period due to the inhibition of moisture entry into the container (Harrington, 1973). In smaller seeds of agricultural and horticultural crops also similar variation in moisture content with relation to container and storage period was reported (Punithavathi, 1997; Menaka, 2000). 
Tompsett (1986) also reported that the storability vary with container and the main cause behind them was the moisture content due to the variation they exert under altering atmosphere to attain the moisture equilibrium status (Agrawal, 1995). Among the seed treatments, the increase in moisture content was non significant in line with Shaw (1998) expressing that slurry seed treatment is not the factor that alters the absorption capacity of the seeds.

The germination and vigour that are viewed as the physiological quality characters of seed, decreased with advances in storage period. In the present study, a progressive decline in germination was observed from 96 to 75 per cent within 12 months, irrespective of treatments and containers. The decline in germination during storage could be attributed to the depletion of food reserves and metabolic activity by Heydecker (1972). Roberts (1972) also coined the causes as the physiological ageing process that had been accelerated by the interactive effect of seed moisture and physiological senescence process (Kovalenko et al., 1977). Natarajan (2001) also opined that such similarity between moisture content and germination with marigold, as evident in the present study. Lowig (1969) and Han (1984) also revealed that the seed treatment, could modify the physiological status of seed during storage. In the present study, germination of seeds declined to 79 per cent, in seeds treated with halogen mixture while the other treatments were in the order of diflubenzuron, bavistin and untreated seed recording 78,74 and 70 per cent germination at the end of the 12 months. Halogen mixture was scored as the best ecofriendly chemical for seed treatment where the protection was found to be due to stabilization of unsaturated fatty acids of double bonds which reduced the formation of free radicals in seeds during storage (Basu, 1994; Dharamalingam et al., 1998;
Malarkodi and Dharmalingam, 1997). Gupta (2001) explained that diflubenzuron is an insect growth regulator that inhibit the chitin synthesis in the insect body and reduce the biotic stress, the seed being experienced during storage and thereby maintained the germination of seed at higher level. In the present study also the next best chemical scored for seed treatment was the diflubenzuron. Similar results were obtained with Sudha et al., (2003) with rice and pearlmillet. With Desmanthus, Reshma (2001) also reported similar results where the insect activity was nil throughout the storage period due to the seed treatment with diflubenzuron. But in the present study,the insect activity was nil throughout the storage period but the chemical could maintain the germination of seed by control of seed deterioration rate which could be due to the unidentified beneficial effect of the chemical to maintain the seed quality during storage in addition to the influence on insect control.

Several scientists expressed that bavistin have the antisenescence factor in addition to the systemic approach towards pathogen control because of which in the present study, though the pathogen infection, even storage fungi was not noticed in this small seed, treatment with bavistin protected the seed during storage from the natural deteriorative senescence.

The vigour of seeds examined in terms of seedling growth, drymatter production and vigour index were also influenced by the evaluated seed quality factors viz., seed treatments, storage containers and storage periods. Seeds stored in aluminium foil polylaminated pouches after treating with halogen mixture recorded the higher values than other chemicals and 700 gauge polyethylene bag were on par with aluminium foil polylaminated pouches and was followed by paper bag (Table 1-6). 
Table.1 Influence of seed treatments, containers and storage periods on moisture content (\%) of seeds

\begin{tabular}{|c|c|c|c|c|c|c|c|c|}
\hline \multirow[t]{2}{*}{ Seed treatments $(\mathrm{T})$} & \multirow{2}{*}{\multicolumn{2}{|c|}{ Storage containers $(\mathrm{C})$}} & \multicolumn{5}{|c|}{ Storage period in months $(\mathrm{P})$} & \multirow[t]{2}{*}{ Mean } \\
\hline & & & 0 & 3 & 6 & 9 & 12 & \\
\hline \multirow[t]{4}{*}{ Untreated } & \multicolumn{2}{|c|}{ Paper bag } & 8.0 & 8.3 & 8.4 & 8.5 & 8.7 & 8.4 \\
\hline & \multicolumn{2}{|c|}{700 gauge poly bag } & 8.0 & 8.0 & 8.0 & 8.0 & 8.2 & 8.0 \\
\hline & \multicolumn{2}{|c|}{ AFLP } & 8.0 & 8.0 & 8.0 & 8.0 & 8.2 & 8.0 \\
\hline & \multicolumn{2}{|c|}{ Mean } & 8.0 & 8.1 & 8.1 & 8.2 & 8.4 & 8.2 \\
\hline \multirow[t]{4}{*}{ Bavistin } & \multicolumn{2}{|c|}{ Paper bag } & 8.0 & 8.2 & 8.2 & 8.3 & 8.6 & 8.3 \\
\hline & \multicolumn{2}{|c|}{700 gauge poly bag } & 8.0 & 8.0 & 8.0 & 8.0 & 8.1 & 8.0 \\
\hline & \multicolumn{2}{|c|}{ AFLP } & 8.0 & 8.0 & 8.0 & 8.0 & 8.1 & 8.0 \\
\hline & \multicolumn{2}{|c|}{ Mean } & 8.0 & 8.1 & 8.1 & 8.1 & 8.3 & 8.1 \\
\hline \multirow[t]{4}{*}{ Halogen } & \multicolumn{2}{|c|}{ Paper bag } & 8.0 & 8.2 & 8.3 & 8.3 & 8.5 & 8.3 \\
\hline & \multicolumn{2}{|c|}{700 gauge poly bag } & 8.0 & 8.0 & 8.0 & 8.0 & 8.2 & 8.0 \\
\hline & \multicolumn{2}{|c|}{ AFLP } & 8.0 & 8.0 & 8.0 & 8.0 & 8.1 & 8.0 \\
\hline & \multicolumn{2}{|c|}{ Mean } & 8.0 & 8.1 & 8.1 & 8.1 & 8.3 & 8.1 \\
\hline \multirow[t]{4}{*}{ Diflubenzuron } & \multicolumn{2}{|c|}{ Paper bag } & 8.0 & 8.2 & 8.2 & 8.4 & 8.5 & 8.3 \\
\hline & \multicolumn{2}{|c|}{700 gauge poly bag } & 8.0 & 8.0 & 8.0 & 8.0 & 8.1 & 8.0 \\
\hline & \multicolumn{2}{|c|}{ Aluminium foil } & 8.0 & 8.0 & 8.0 & 8.0 & 8.1 & 8.0 \\
\hline & \multicolumn{2}{|c|}{ Mean } & 8.0 & 8.1 & 8.1 & 8.1 & 8.2 & 8.1 \\
\hline \multirow[t]{4}{*}{$\mathrm{C} \times \mathrm{P}$} & \multicolumn{2}{|c|}{ Paper bag } & 8.0 & 8.2 & 8.3 & 8.4 & 8.6 & 8.3 \\
\hline & \multicolumn{2}{|c|}{700 gauge poly bag } & 8.0 & 8.0 & 8.0 & 8.0 & 8.2 & 8.0 \\
\hline & \multicolumn{2}{|c|}{ Aluminium foil } & 8.0 & 8.0 & 8.0 & 8.0 & 8.1 & 8.0 \\
\hline & \multicolumn{2}{|c|}{ Mean } & 8.0 & 8.1 & 8.1 & 8.1 & 8.3 & 8.1 \\
\hline \multirow[t]{2}{*}{$\mathrm{CD}(\mathrm{P}=0.05)$} & $\mathrm{T}$ & $\mathrm{P}$ & C & TP & & & & TPC \\
\hline & NS & 0.139 & 0.108 & NS & & & & NS \\
\hline
\end{tabular}

Table. 2 Influence of seed treatments, containers and storage periods on germination (\%) of seeds

\begin{tabular}{|c|c|c|c|c|c|c|c|c|}
\hline \multirow{2}{*}{$\begin{array}{l}\text { Seed treatments } \\
\text { (T) }\end{array}$} & \multirow{2}{*}{\multicolumn{2}{|c|}{ Storage containers $(\mathrm{C})$}} & \multicolumn{5}{|c|}{ Storage period in months $(\mathrm{P})$} & \multirow[t]{2}{*}{ Mean } \\
\hline & & & 0 & 3 & 6 & 9 & 12 & \\
\hline \multirow[t]{4}{*}{ Untreated } & \multicolumn{2}{|l|}{ Paper bag } & $96(79.13)$ & $90(71.62)$ & $85(67.28)$ & 79 (62.76) & $68(55.57)$ & $84(67.27)$ \\
\hline & \multicolumn{2}{|c|}{700 gauge poly bag } & 96 (79.13) & $90(71.58)$ & $87(68.87)$ & $82(64.91)$ & $71(57.42)$ & $85(68.38)$ \\
\hline & \multicolumn{2}{|c|}{ Aluminium foil } & 96 (79.13) & $91(72.56)$ & $88(69.77)$ & $83(65.67)$ & $72(58.07)$ & $86(69.04)$ \\
\hline & \multicolumn{2}{|l|}{ Mean } & 96 (79.13) & $90(71.92)$ & 87 (68.64) & $81(64.45)$ & $70(57.02)$ & 85 (68.23) \\
\hline \multirow[t]{4}{*}{ Bavistin } & \multicolumn{2}{|l|}{ Paper bag } & $96(79.13)$ & $91(73.04)$ & $86(68.06)$ & $80(63.50)$ & $72(58.07)$ & $85(68.36)$ \\
\hline & \multicolumn{2}{|c|}{700 gauge poly bag } & $96(79.13)$ & $92(73.65)$ & $89(70.68)$ & $83(65.70)$ & $74(59.38)$ & 87 (69.71) \\
\hline & \multicolumn{2}{|c|}{ Aluminium foil } & 96 (79.13) & $92(73.65)$ & $89(70.83)$ & $84(66.45)$ & 77 (61.37) & $88(70.29)$ \\
\hline & Mean & & 96 (79.13) & $92(73.45)$ & $88(69.86)$ & $82(65.22)$ & $74(59.61)$ & 87 (69.45) \\
\hline Halogen & Paper bag & & 96 (79.13) & $92(73.76)$ & $88(69.74)$ & $82(64.90)$ & $77(61.38)$ & $87(69.56)$ \\
\hline & 700 gauge & & 96 (79.13) & $93(74.68)$ & $91(72.56)$ & $85(67.40)$ & $78(62.04)$ & 89 (70.94) \\
\hline & Aluminiu & & 96 (79.13) & $93(74.89)$ & $90(71.62)$ & 88 (69.91) & $82(64.90)$ & $90(71.86)$ \\
\hline & Mean & & $96(79.13)$ & $93(74.44)$ & $90(71.31)$ & $85(67.40)$ & 79 (62.77) & 88 (70.79) \\
\hline Diflubenzuron & Paper bag & & $96(79.13)$ & $92(73.65)$ & $87(68.95)$ & $81(64.16)$ & $76(60.67)$ & $86(69.19)$ \\
\hline & 700 gauge & & 96 (79.13) & 93 (74.76) & $90(71.80)$ & 87 (69.24) & 77 (61.37) & 89 (71.14) \\
\hline & Aluminiu & & 96 (79.13) & $93(74.89)$ & $90(71.62)$ & 89 (70.74) & $81(64.23)$ & $90(72.00)$ \\
\hline & Mean & & 96 (79.13) & 93 (74.43) & 89 (70.79) & $86(68.05)$ & $78(62.09)$ & 88 (70.79) \\
\hline $\mathrm{C} \times \mathrm{P}$ & Paper bag & & $96(79.13)$ & $91(73.20)$ & $87(68.51)$ & $81(63.83)$ & $73(58.92)$ & $86(68.59)$ \\
\hline & 700 gauge & & 96 (79.13) & $92(73.67)$ & 89 (70.98) & $84(66.81)$ & $75(60.05)$ & 87 (70.04) \\
\hline & Aluminiu & & 96 (79.13) & $92(74.00)$ & 89 (70.96) & $86(68.19)$ & $78(62.14)$ & $88(70.80)$ \\
\hline & Mean & & $96(79.13)$ & $92(73.56)$ & 88 (70.15) & $84(66.28)$ & $75(60.37)$ & 87 (69.82) \\
\hline $\mathrm{CD}(\mathrm{P}=0.05)$ & $\mathrm{T}$ & $P$ & $\mathrm{C}$ & TP & PC & & & TPC \\
\hline & 0.917 & 1.025 & 0.794 & 2.051 & 1.7 & & & NS \\
\hline
\end{tabular}

(Figures in parentheses indicate arc sine values) 
Table.3 Influence of seed treatments, containers and storage periods on root length $(\mathrm{cm})$ of seedlings

\begin{tabular}{|c|c|c|c|c|c|c|c|c|}
\hline \multirow[t]{2}{*}{ Seed treatments $(\mathrm{T})$} & \multirow{2}{*}{\multicolumn{2}{|c|}{ Storage containers $(\mathrm{C})$}} & \multicolumn{5}{|c|}{ Storage period in months $(\mathrm{P})$} & \multirow[t]{2}{*}{ Mean } \\
\hline & & & 0 & 3 & 6 & 9 & 12 & \\
\hline \multirow[t]{4}{*}{ Untreated } & \multicolumn{2}{|c|}{ Paper bag } & 0.9 & 0.8 & 0.7 & 0.5 & 0.3 & 0.6 \\
\hline & \multicolumn{2}{|c|}{700 gauge poly bag } & 0.9 & 0.8 & 0.8 & 0.6 & 0.4 & 0.7 \\
\hline & \multicolumn{2}{|c|}{ Aluminium foil } & 0.9 & 0.8 & 0.8 & 0.6 & 0.4 & 0.7 \\
\hline & \multicolumn{2}{|c|}{ Mean } & 0.9 & 0.8 & 0.8 & 0.6 & 0.4 & 0.7 \\
\hline \multirow[t]{4}{*}{ Bavistin } & \multicolumn{2}{|c|}{ Paper bag } & 0.9 & 0.9 & 0.7 & 0.6 & 0.5 & 0.7 \\
\hline & \multicolumn{2}{|c|}{700 gauge poly bag } & 0.9 & 0.9 & 0.9 & 0.7 & 0.6 & 0.8 \\
\hline & \multicolumn{2}{|c|}{ Aluminium foil } & 0.9 & 0.9 & 0.9 & 0.7 & 0.6 & 0.8 \\
\hline & \multicolumn{2}{|c|}{ Mean } & 0.9 & 0.9 & 0.8 & 0.7 & 0.6 & 0.8 \\
\hline \multirow[t]{4}{*}{ Halogen } & \multicolumn{2}{|c|}{ Paper bag } & 1.0 & 0.9 & 0.8 & 0.7 & 0.6 & 0.8 \\
\hline & \multicolumn{2}{|c|}{700 gauge poly bag } & 1.0 & 0.9 & 0.9 & 0.8 & 0.7 & 0.9 \\
\hline & \multicolumn{2}{|c|}{ Aluminium foil } & 1.0 & 0.9 & 0.9 & 0.8 & 0.7 & 0.9 \\
\hline & \multicolumn{2}{|c|}{ Mean } & 1.0 & 0.9 & 0.9 & 0.8 & 0.7 & 0.8 \\
\hline \multirow[t]{4}{*}{ Diflubenzuron } & \multicolumn{2}{|c|}{ Paper bag } & 1.0 & 0.7 & 0.8 & 0.9 & 0.6 & 0.8 \\
\hline & \multicolumn{2}{|c|}{700 gauge poly bag } & 1.0 & 1.0 & 0.9 & 0.7 & 0.7 & 0.8 \\
\hline & \multicolumn{2}{|c|}{ Aluminium foil } & 1.0 & 1.0 & 0.9 & 0.8 & 0.7 & 0.9 \\
\hline & \multicolumn{2}{|c|}{ Mean } & 1.0 & 0.9 & 0.8 & 0.7 & 0.7 & 0.9 \\
\hline \multirow[t]{4}{*}{$\mathrm{C} \times \mathrm{P}$} & \multicolumn{2}{|c|}{ Paper bag } & 1.0 & 0.8 & 0.7 & 0.7 & 0.6 & 0.8 \\
\hline & \multicolumn{2}{|c|}{700 gauge poly bag } & 1.0 & 0.9 & 0.9 & 0.7 & 0.6 & 0.8 \\
\hline & \multicolumn{2}{|c|}{ Aluminium foil } & 1.0 & 0.9 & 0.9 & 0.7 & 0.6 & 0.8 \\
\hline & \multicolumn{2}{|c|}{ Mean } & 1.0 & 0.9 & 0.8 & 0.7 & 0.6 & 0.8 \\
\hline \multirow[t]{2}{*}{$\mathrm{CD}(\mathrm{P}=0.05)$} & $\mathrm{T}$ & $\mathrm{P}$ & $\mathrm{C}$ & TP & PC & & & ТPC \\
\hline & 0.068 & 0.076 & NS & NS & NS & & & NS \\
\hline
\end{tabular}

Table.4 Influence of seed treatments, containers and storage periods on shoot length $(\mathrm{cm})$ of seedlings

\begin{tabular}{|c|c|c|c|c|c|c|c|}
\hline \multirow{2}{*}{$\begin{array}{l}\text { Seed treatments } \\
\text { (T) }\end{array}$} & \multirow[t]{2}{*}{ Storage containers $(\mathrm{C})$} & \multicolumn{5}{|c|}{ Storage period in months $(\mathbf{P})$} & \multirow[t]{2}{*}{ Mean } \\
\hline & & 0 & 3 & 6 & 9 & 12 & \\
\hline \multirow[t]{4}{*}{ Untreated } & Paper bag & 1.9 & 1.7 & 1.7 & 1.3 & 1.2 & 1.6 \\
\hline & 700 gauge poly bag & 1.9 & 1.8 & 1.8 & 1.4 & 1.4 & 1.7 \\
\hline & Aluminium foil & 1.9 & 1.8 & 1.8 & 1.4 & 1.5 & 1.7 \\
\hline & Mean & 1.9 & 1.8 & 1.8 & 1.4 & 1.4 & 1.6 \\
\hline \multirow[t]{4}{*}{ Bavistin } & Paper bag & 2.0 & 1.9 & 1.8 & 1.6 & 1.4 & 1.7 \\
\hline & 700 gauge poly bag & 2.0 & 2.0 & 1.9 & 1.7 & 1.5 & 1.8 \\
\hline & Aluminium foil & 2.0 & 1.9 & 1.9 & 1.7 & 1.6 & 1.8 \\
\hline & Mean & 2.0 & 1.9 & 1.9 & 1.7 & 1.5 & 1.8 \\
\hline \multirow[t]{4}{*}{ Halogen } & Paper bag & 2.0 & 1.9 & 1.8 & 1.7 & 1.6 & 1.8 \\
\hline & 700 gauge poly bag & 2.0 & 1.9 & 1.9 & 1.8 & 1.8 & 1.9 \\
\hline & Aluminium foil & 2.0 & 2.0 & 1.9 & 1.8 & 1.9 & 1.9 \\
\hline & Mean & 2.0 & 1.9 & 1.9 & 1.8 & 1.8 & 1.9 \\
\hline \multirow[t]{4}{*}{ Diflubenzuron } & Paper bag & 2.1 & 1.9 & 1.8 & 1.7 & 1.7 & 1.8 \\
\hline & 700 gauge poly bag & 2.1 & 2.0 & 1.9 & 1.8 & 1.8 & 1.9 \\
\hline & Aluminium foil & 2.1 & 2.0 & 1.9 & 1.8 & 1.8 & 1.9 \\
\hline & Mean & 2.1 & 2.0 & 1.9 & 1.8 & 1.8 & 1.9 \\
\hline \multirow[t]{4}{*}{$\mathbf{C} \times \mathbf{P}$} & Paper bag & 2.0 & 1.9 & 1.8 & 1.6 & 1.5 & 1.7 \\
\hline & 700 gauge poly bag & 2.0 & 1.9 & 1.9 & 1.7 & 1.6 & 1.8 \\
\hline & Aluminium foil & 2.0 & 1.9 & 1.8 & 1.7 & 1.7 & 1.8 \\
\hline & Mean & 2.0 & 1.9 & 1.8 & 1.6 & 1.6 & 1.8 \\
\hline \multirow[t]{2}{*}{ CD $(P=0.05)$} & $\mathrm{T}$ & $\mathrm{C}$ & TP & & & $\mathrm{TC}$ & TPC \\
\hline & 0.091 & 0.070 & NS & & & NS & NS \\
\hline
\end{tabular}


Table.5 Influence of seed treatments, containers and storage periods on drymatter production (mg 20 seedlings-1)

\begin{tabular}{|c|c|c|c|c|c|c|c|c|}
\hline \multirow{2}{*}{$\begin{array}{l}\text { Seed treatments } \\
\text { (T) }\end{array}$} & \multirow{2}{*}{\multicolumn{2}{|c|}{ Storage containers $(C)$}} & \multicolumn{5}{|c|}{ Storage period in months $(\mathbf{P})$} & \multirow[t]{2}{*}{ Mean } \\
\hline & & & 0 & 3 & 6 & 9 & 12 & \\
\hline \multirow[t]{4}{*}{ Untreated } & \multicolumn{2}{|l|}{ Paper bag } & 1.9 & 1.8 & 1.7 & 1.5 & 1.4 & 1.7 \\
\hline & \multicolumn{2}{|c|}{700 gauge poly bag } & 1.9 & 1.9 & 1.8 & 1.6 & 1.5 & 1.7 \\
\hline & \multicolumn{2}{|c|}{ Aluminium foil } & 1.9 & 1.9 & 1.8 & 1.7 & 1.5 & 1.8 \\
\hline & \multicolumn{2}{|c|}{ Mean } & 1.9 & 1.9 & 1.8 & 1.6 & 1.5 & 1.7 \\
\hline \multirow[t]{4}{*}{ Bavistin } & \multicolumn{2}{|l|}{ Paper bag } & 2.0 & 1.9 & 1.8 & 1.6 & 1.5 & 1.8 \\
\hline & \multicolumn{2}{|c|}{700 gauge poly bag } & 2.0 & 2.0 & 1.9 & 1.8 & 1.6 & 1.9 \\
\hline & \multicolumn{2}{|c|}{ Aluminium foil } & 2.0 & 2.0 & 1.9 & 1.8 & 1.6 & 1.9 \\
\hline & \multicolumn{2}{|l|}{ Mean } & 2.0 & 2.0 & 1.9 & 1.7 & 1.6 & 1.8 \\
\hline \multirow[t]{4}{*}{ Halogen } & \multicolumn{2}{|l|}{ Paper bag } & 2.1 & 2.1 & 1.9 & 1.7 & 1.6 & 1.9 \\
\hline & \multicolumn{2}{|c|}{700 gauge poly bag } & 2.1 & 2.1 & 2.0 & 1.8 & 1.7 & 1.9 \\
\hline & \multicolumn{2}{|c|}{ Aluminium foil } & 2.1 & 21 & 2.0 & 1.9 & 1.7 & 2.0 \\
\hline & \multicolumn{2}{|c|}{ Mean } & 2.1 & 2.1 & 2.0 & 1.8 & 1.7 & 1.9 \\
\hline \multirow[t]{4}{*}{ Diflubenzuron } & \multicolumn{2}{|l|}{ Paper bag } & 2.2 & 2.0 & 1.9 & 1.7 & 1.6 & 1.9 \\
\hline & \multicolumn{2}{|c|}{700 gauge poly bag } & 2.2 & 2.1 & 2.0 & 1.8 & 1.8 & 2.0 \\
\hline & \multicolumn{2}{|c|}{ Aluminium foil } & 2.2 & 2.1 & 2.0 & 1.9 & 1.7 & 2.0 \\
\hline & \multicolumn{2}{|c|}{ Mean } & 2.2 & 2.1 & 2.0 & 1.8 & 1.7 & 1.9 \\
\hline \multirow[t]{4}{*}{$\mathbf{C} \times \mathbf{P}$} & \multicolumn{2}{|l|}{ Paper bag } & 2.0 & 2.0 & 1.8 & 1.6 & 1.5 & 1.8 \\
\hline & \multicolumn{2}{|c|}{700 gauge poly bag } & 2.0 & 2.0 & 1.9 & 1.8 & 1.7 & 1.9 \\
\hline & \multicolumn{2}{|c|}{ Aluminium foil } & 2.0 & 2.0 & 1.9 & 1.8 & 1.6 & 1.9 \\
\hline & \multicolumn{2}{|l|}{ Mean } & 2.0 & 2.0 & 1.9 & 1.7 & 1.6 & 1.9 \\
\hline \multirow[t]{2}{*}{$\mathrm{CD}(\mathrm{P}=0.05)$} & $\mathrm{T}$ & $\mathrm{P}$ & $\mathrm{C}$ & & & & TC & TPC \\
\hline & 0.077 & 0.086 & 0.067 & & & & NS & NS \\
\hline
\end{tabular}

Table.6 Influence of seed treatments, containers and storage periods on vigour index

\begin{tabular}{|c|c|c|c|c|c|c|c|c|}
\hline \multirow{2}{*}{$\begin{array}{l}\text { Seed treatments } \\
(\mathrm{T})\end{array}$} & \multirow{2}{*}{\multicolumn{2}{|c|}{ Storage containers $(\mathrm{C})$}} & \multicolumn{5}{|c|}{ Storage period in months $(\mathbf{P})$} & \multirow[t]{2}{*}{ Mean } \\
\hline & & & 0 & 3 & 6 & 9 & 12 & \\
\hline \multirow[t]{4}{*}{ Untreated } & \multicolumn{2}{|l|}{ Paper bag } & 183 & 168 & 147 & 119 & 96 & 142 \\
\hline & \multicolumn{2}{|c|}{700 gauge poly bag } & 183 & 171 & 154 & 131 & 107 & 149 \\
\hline & \multicolumn{2}{|c|}{ Aluminium foil } & 183 & 170 & 159 & 141 & 108 & 152 \\
\hline & \multicolumn{2}{|c|}{ Mean } & 183 & 170 & 153 & 130 & 104 & 148 \\
\hline \multirow[t]{4}{*}{ Bavistin } & \multicolumn{2}{|l|}{ Paper bag } & 193 & 174 & 155 & 128 & 108 & 152 \\
\hline & \multicolumn{2}{|c|}{700 gauge poly bag } & 193 & 184 & 169 & 149 & 119 & 163 \\
\hline & \multicolumn{2}{|c|}{ Aluminium foil } & 193 & 184 & 170 & 151 & 124 & 164 \\
\hline & \multicolumn{2}{|l|}{ Mean } & 193 & 181 & 165 & 143 & 117 & 160 \\
\hline \multirow[t]{4}{*}{ Halogen } & \multicolumn{2}{|l|}{ Paper bag } & 204 & 195 & 167 & 139 & 124 & 166 \\
\hline & \multicolumn{2}{|c|}{700 gauge poly bag } & 204 & 195 & 182 & 153 & 133 & 174 \\
\hline & \multicolumn{2}{|c|}{ Aluminium foil } & 204 & 195 & 180 & 161 & 139 & 176 \\
\hline & \multicolumn{2}{|c|}{ Mean } & 204 & 195 & 177 & 151 & 132 & 172 \\
\hline \multirow[t]{4}{*}{ Diflubenzuron } & \multicolumn{2}{|l|}{ Paper bag } & 211 & 184 & 166 & 138 & 122 & 164 \\
\hline & \multicolumn{2}{|c|}{700 gauge poly bag } & 211 & 195 & 181 & 151 & 139 & 175 \\
\hline & \multicolumn{2}{|c|}{ Aluminium foil } & 211 & 195 & 180 & 161 & 138 & 177 \\
\hline & \multicolumn{2}{|l|}{ Mean } & 211 & 192 & 175 & 150 & 133 & 172 \\
\hline \multirow[t]{4}{*}{$\mathbf{C} \times \mathbf{P}$} & \multicolumn{2}{|l|}{ Paper bag } & 198 & 180 & 159 & 131 & 112 & 156 \\
\hline & \multicolumn{2}{|c|}{700 gauge poly bag } & 198 & 186 & 172 & 146 & 124 & 165 \\
\hline & \multicolumn{2}{|c|}{ Aluminium foil } & 198 & 186 & 172 & 154 & 127 & 167 \\
\hline & \multicolumn{2}{|l|}{ Mean } & 198 & 184 & 167 & 144 & 121 & 163 \\
\hline \multirow[t]{2}{*}{$\mathrm{CD}(\mathrm{P}=0.05)$} & $\mathrm{T}$ & $\mathrm{P}$ & $\mathrm{C}$ & & & & TC & TPC \\
\hline & 7.544 & 8.435 & 6.534 & & & & NS & NS \\
\hline
\end{tabular}

Irrespective of seed treatments and storage period, the aluminium foil polylaminated pouches excelled other containers in maintenance of seed germination during 
storage due to its moisture vapour proof nature. As expressed by Srimathi et al., (2001) it could have offered protection against invasion of pathogen and insects by restricting the fluctuation in the moisture content of the seeds due to atmospheric alterations as detailed elsewhere. Charjan and Tarar (1991) in sesame, Sathyanarayanan (2000) in phlox, Natarajan (2000) in marigold and Vijayan (2002) in zinnia also opined similar results of lesser reduction in storability of seeds in moisture vapour proof container than moisture previous container. The germination of seeds stored in 700 gauge polyethylene bag was similar to that of aluminium foil polylaminated pouch as their relation to permissibility to moisture content from outside to inside and vice versa are completely similar to one another.

The reduction in physiological stamina of seeds could be due to complex environment and genetic factors influencing the potentiality of the seed. Arunachalam (1991) in balsam, Natarajan (2000) in marigold and Vijayan (2002) in zinnia and gaillardia also observed similar results. With advances in storage period, irrespective of treatments and containers the vigour parameters reduced by 40 per cent for root length, 20 per cent for shoot length and drymatter production and 39 per cent for vigour index over 12 months of storage compared to initial value of seed storage. This decrease was also supported by Sathiyanarayanan (2000) and Vijayan (2002) in various small seeded flower crops. Thus the present study indicated that seeds received with storage management practices could mitigate the deterioration rate. Thus the study highlighted that

The germination reduces from 96 to 75 per cent with 12 months of storage period under ambient condition
Among the seed treatments, halogen mixture followed by diflubenzuron found to be the better as seed treating chemicals.

Storability of seed is better in moisture vapour proof container (aluminium foiled pouch) than moisture pervious container (paper bags).

\section{References}

Abdul-Baki, A.A. and J.D. Anderson. 1973. Vigour determination in soybean seed by multiple criteria. Crop Sci., 13: 630-633.

Agrawal, R.L. 1995. Seed Technology. Oxford and IBH Publishing Co., Pvt. Ltd., New Delhi.

Arunachalam, M. 1991. Studies on seed development, maturation and pattern of deterioration of seeds of six genotypes of Balsam. M.Sc. (Ag.) Thesis, Tamil Nadu Agricultural University, Coimbatore.

Barros, B.C., C.L. Salgado and C.C. Lasca. 1983. Action of fungicides in vitro on the germination and mycoflora of wheat seeds. Summa Phytopathoogical, 9(1/2): 118-127.

Basu, R.N. 1994. An appraisal of research o wet and dry physiological treatments and their applicability with special reference to tropical and subtropical countries. Seed Sci \& Technol., 22: 107-126.

Basu, R.N. and A.B. Rudrapal. 1980. Iodination of mustard seed for the maintenance of vigour and viability. Indian J. Exp. Biol., 18: 491-494.

Bhattacharya, P., R.C. Samui and S. Sen. 1982. Studies on the germination and viability of stored sunflower seed. Seed Res., 11; 171.

Burris, J.S. 2002. The impact of thiamethoxan seed treatment on maize storability and laboratory test performance. Seed Abstr., 13: 1871.

Charjan, S.K.U. and J.L. Tarar. 1991. Storability of sesame (Sesamum indicum L.) seed as influenced by storage containers. Ann. Plant Physiol., 5(2): 160-165.

Copeland, L.O. 1988. Seed deterioration. In: Principles of Seed Science and Technology, Subject Publishers, New Delhi, pp. 107115. 
Copeland, L.O. and M.B. Mcdonald, 1995. Principles of Seed Science and Technology, Mac Millan Publishing Company, Newyork.

Delouche, J.C. and C.C. Baskin. 1973. Accelerated ageing technique for predicting the relative storability of seed lots. Seed Sci. \& Technol., 1: 427-452.

Dharmalingam, C., R.Vasantha, K. Malarkodi and S. Lakshmi. 2000. Halogenation treatment to safeguard pulse seeds in storage under ambient condition. Seed Res., 28(1): 42-46.

Dharmalingam, C., S. Lakshmi, K., Malarkodi and S.K. Ananathi. 1998. Halogenation to shelf life of seeds. Department of Seed Science and Technology, Tamil Nadu Agricultural University, Coimbatore.

Elek, J.A. and B.C. Longstaff. 1994. Effect of chitin-synthesis inhibitors on stored product beetles. Pestic. Sci., 40: 225-230.

Gomez, K.A. and A.A. Gomez. 1984. Statistical procedures for agricultural research of legumes seeds of different density. Agriculture, 41:47-56

Gupta, H.C. L. 2001. In: Insecticides: Toxicology and uses. Agrotech Publishing Academy, Udaipur, pp: 185-210.

Han, N.1. 1984. Studies on the technique of lowtemperature storage of seeds of Camellia oleifera. Forest Sci. Technol., 12: 7-9.

Harrrington, J.K. 1973. Biochemical basis of seed longevity. Seed Sci. \& Technol., 1: 453461.

Heydecker, W. 1972. Vigour. In: Viability of seeds (ed. E.H. Roberts), chapman and Hall London, pp. 209-252.

ISTA. 1999. International Rules for Seed Testing. Seed Sci. \& Technol. (Supplement Rules), 27: 25-30.

Justice, O.L. and L.N. Bass. 1978. Principles and practices of seed storage. (ed. Oran L. Justice and Louis N. Bass). Printing office, Washington.

Kovalenko, G.I., D.Badev, and R.A. Falik.1977. Some aspects of germination loss of cotton seeds. Biologiche Kishnask, 6: 26-30.

Lowig, E. 1969. The storage property of petunia seeds. Saatgut Wirtsch, 21: 28.

Malarkodi, K. and C. Dharmalingam. 1997. Comparative efficacy of seed treatments to control seed deterioration in bajra seed lots of different seed quality. Neo Botanica 5(1\&2): 55-61.

Marnaville, J.H. and M.D. Clegg. 1977. Influence of seed size and density on germination, seedling emergence and yield of grain sorghum. Agron. J., 69: 329-330.

Menaka, C. 2000. Seed technological studies in amaranthus (Amaranthus tricolor) cv. CO 5. M.Sc. (Ag.) Thesis, Tamil Nadu Agricultural University, Coimbatore.

Natarajan, K. 2000. Seed technological studies in marigold (Tagetes erecta). M.Sc.(Ag.) Thesis, Tamil Nadu Agricultural University, Coimbatore.

Osborne, D.J. 1980. In: Senescence in plants. (Ed. Thimann. K.V.). CRC. Press. pp. 13-37.

Punithavathi, N. 1997. Seed hardening and pelleting studies in ragi cv. CO5. M.Sc. (Ag.) Thesis, Tamil Nadu Agricultural University, Coimbatore.

Reshma, C. 2001. Studies on maturation, production, processing and storage in hedge Lucerne cv. TNDV1. M.Sc.(Ag.) Thesis, Tamil Nadu Agricultural University, Coimbatore.

Roberts, E.H. 1972. Loss of viability and crop yields. In: Viability of Seeds (ed. E.H. Roberts), Chapman and Hall Ltd., London, p. 313.

Roberts, E.H. 1986. Quantifying seed deterioration. In: Physiology of seed deterioration (eds. M.B. McDonals Jr. and C.J. Nelson). Crop Science Society America Special Publication Number 11, CSSA, Madison, WI, pp. 101-123.

Sathiyanarayanan, G. 2000. Seed technological studies in phlox. M.Sc.(Ag.) Thesis, Tamil Nadu Agricultural University, Coimbatore.

Shaw, K.K. 1998. Some quantitative and qualitative effects on greengram stored under conditions of different initial grain moisture contents, storage periods and storage containers. J. Agric. Sci. Society of Northeast India, 11(1): 61-65.

Srimathi, P., K. Malarkodi and G. Sasthri. 2001. Influence of fungicides and insecticides on storability pearlmillet seed. Madras Agric J., 88(1-3): 16-21.

Sudha, P., P. Srimathi and K. Vanangamudi. 2003. Storability of paddy seeds as 
influenced by insecticidal and fungicidal seed treatments. Paper presented in National level Seminar on"Global strategies in biological sciences " conducted by Muthyammal college of arts and science, Rasipuram. Pp-65.

Sudha, R. 2002. Influence of pesticidal treatments on cereal seed storage. M.Sc. (Ag.) Thesis, Tamil Nadu Agricultural University,
Coimbatore.

Tompsett, P.B. 1986. The effect of desiccation on the viability of dipterocarpous seed. Fedual Forest Institute, Report No. 12, Vienna, pp. 181-202.

Vijayan, R. 2002. Seed hardening and conditioning in Zinnia. M.Sc.(Ag.) Thesis, Tamil Nadu Agricultural University, Coimbatore.

\section{How to cite this article:}

Natarajan, K. 2019. Seed Treatments and Storage Containers on Storability of Petunia Seeds. Int.J.Curr.Microbiol.App.Sci. 8(06): 1106-1114. doi: https://doi.org/10.20546/ijcmas.2019.806.137 